\title{
UNA EXPERIENCIA INTERGENERACIONAL DE APRENDIZAJE-SERVICIO EN LA FORMACIÓN PROFESIONAL EN EXTREMADURA: \#apdintergeneracional"
}

\author{
AN INTERGENERATIONAL SERVICE-LEARNING EXPERIENCE IN \\ VOCATIONAL TRAINING IN EXTREMADURA: "\#apdintergeneracional
}

\author{
Mª Gloria Solís Galán*, Mª José Galán Gamonales** \\ Universidad de Extremadura*, IES Al-Qázeres (Cáceres)**
}

\author{
Correspondencia: Ma Gloria Solís Galán \\ Correo: glsolisg@unex.es \\ Recibido: 29-03-2020; Aceptado: 09/06/2020 \\ DOI: $10.17398 / 0213-9529.40 .1 .45$
}

\begin{abstract}
RESUMEN
El presente artículo describe un proyecto de innovación educativa en la Formación Profesional a través de la metodología de Aprendizaje-Servicio (ApS). Se lleva a cabo en el ciclo de Grado Medio de Atención a Personas en Situación de Dependencia, durante los cursos 2017-2018 y 2019-2020. Surge de la necesidad de eliminar estereotipos y empatizar con el colectivo de personas mayores. Desde esta premisa se inicia un proceso de reflexión a través de herramientas de Diagnóstico Social Participativo que derivó en el diseño de un proyecto de ApS con en el alumnado como protagonista activo y agente de transformación. Como instrumento de evaluación se seleccionó la "Rúbrica para la autoevaluación de proyectos de aprendizajeservicio", adaptada a una versión electrónica a través de la aplicación Corubrics. Entre los resultados obtenidos, se apuntan los puntos fuertes y débiles identificados para favorecer la reflexión e implementación de propuestas de mejora o posibilidades de replicabilidad.
\end{abstract}

Palabras clave: Aprendizaje-Servicio; formación profesional; competencias; intergeneracionalidad; innovación educativa.

\begin{abstract}
This article describes an educational innovation project in Vocational Training through the Service-Learning (S-L) methodology. It is carried out in the Intermediate Vocational Training Course of Attention to People in a Situation of Dependency, during the academic years 2017-2018 and 2019-2020. It arises from the need to eliminate stereotypes and empathize with the older people group. From this premise, a process of reflection begins through Participatory Social Diagnosis tools that led to the design of an ApS project with students as an active protagonist and agent of transformation. The "Rubric for self-evaluation of servicelearning projects" was selected as evaluation instrument and was adapted to electronic version through the Corubrics application. Among the results obtained, the identified strong and weak points are pointed out to favor the reflection and implementation of improvement proposals or replicability possibilities.
\end{abstract}

Keyword: Service-Learning; vocational training; competencies; intergenerationality; Innovative education.

Sección I Section: El Aprendizaje Servicio (ApS) en la sociedad actual

Editor de Sección / Edited by:,Ana López Medialdea - Nieves Martin Bermúdez / Universidad de Extremadura, España

Campo Abierto, v. 40, n. 1, p. 45-60, 2021 


\section{INTRODUCCIÓN}

De acuerdo con la legislación educativa vigente: "La Formación Profesional promoverá la integración de contenidos científicos, tecnológicos y organizativos del ámbito profesional, así como los de las materias instrumentales, y garantizará que el alumnado adquiera y amplíe las competencias necesarias para su desarrollo profesional, personal y social (LOMCE, 2013, artículo 42.3). Por tanto, pueden considerarse competencias clave ligadas íntimamente a la Formación Profesional (en adelante FP): 1) aprender a aprender, 2) competencias sociales y cívicas y 3) el sentido de la iniciativa y espíritu emprendedor (Winterton, Delamare-Le Deist y Stringfellow, 2006; citado en Martín, 2015). En este sentido, la FP es considerada una propuesta formativa caracterizada por su flexibilidad y capacidad de adaptación, cualidades necesarias en la sociedad actual "para encajar en la sociedad de hoy y para actuar como motor de cambio y mejora social, en una relación recíproca de continuo intercambio" (Marhuenda, 2012; citado en Martín, 2015, p. 50). Así, en un contexto social cada vez más inestable, se requiere orientar la formación hacia el desarrollo de competencias profesionales específicas para el desempeño laboral en un puesto de trabajo concreto y competencias sociales y personales para el ejercicio de una ciudadanía activa (Tejada, 2013; citado en Martín, 2015). Es por ello que la formación para el empleo debe unirse a la formación para una ciudadanía activa y el desarrollo social, contribuyendo a definir perfiles profesionales multidimensionales que integren las dimensiones profesionalizadora, social, ética y cívica (Tejada, 2013). Estas orientaciones, además, deben interpretarse en el marco de acción mundial que aporta la Agenda 2030 para el Desarrollo Sostenible y lo que ha venido a llamarse Educación para el Desarrollo Sostenible y la Ciudadanía Mundial (UNESCO, 2016, 2017).

Es en tipo de enseñanza, la FP reglada, desde la que se encuadra el presente proyecto educativo del Departamento de Servicios Socioculturales y a la Comunidad (SSC) del IES AlQázeres. En relación con la población objeto de intervención educativa, el colectivo-clase al que se dirigió esta experiencia, pertenecen al grupo de personas jóvenes de entre 16 y 25 años que se encuentra en la zona de vulnerabilidad de exclusión social (Castel, 1997). Más aún, haciendo una lectura intergeneracional, se trata de un proyecto educativo que media entre dos colectivos vulnerables: personas jóvenes y generaciones de mayor edad como posible colectivo de discriminación por las situaciones de dependencia que los afecta (FOESSA, 2019). En definitiva, el Departamento se marca como objetivo diseñar un programa formativo orientado a la adquisición de las competencias curriculares y el desarrollo integral del alumnado, a través de acciones formativas motivadoras que mejoren su rendimiento escolar y garanticen su éxito educativo y social como pueden ser las experiencias inclusivas de intercambio intergeneracional (Flores, Ortega y Vallejo, 2019; Salazar y Castro, 2019).

En esta línea, la metodología de Aprendizaje-Servicio (en adelante ApS) está siendo, cada vez más, considerada como "el recurso más útil para conseguir un aprendizaje significativo de la persona que estudia en esta etapa educativa" (Mateos, Cerrillo y Tello, 2018). Esta tendencia creciente se apoya en las evidencias mostradas por investigaciones educativas centradas en su aplicación en distintas etapas y modalidades educativas, las cuales han demostrado los beneficios de esta filosofía y metodología educativa tanto para el propio alumnado como para el contexto social (Martínez, 2014; Traver, Sales y Moliner, 2018). Se habla de ApS entendiéndose como "una metodología enseñanza-aprendizaje que permite al alumnado implicado desarrollar conocimientos y competencia mediante la prestación de un servicio a colectivos en riesgo de exclusión social" (Tapia, 2008; citado en Chiva y Pallarés, 2018, p.11). Existen otras muchas definiciones de ApS que aportan distintos matices de la misma, pero, en el presente trabajo y 
debido a su proximidad con la orientación a la experiencia que presentamos, recogemos esta conceptualización por razones prácticas.

En la literatura encontramos una falta de investigaciones en relación a la FP (Echevarría y Martínez, 2018, 2019), así como cierta limitación en cuanto a investigaciones centradas en experiencias intergeneracionales, aunque se muestra en expansión (Flores, Ortega y Vallejo, 2019; Salazar y Castro, 2019). A pesar de ello, en lo que respecta al ApS, los méritos de su aplicación educativa están extensamente referidos y documentados como ya hemos referenciado. Así, por ejemplo, Martínez (2014, p.191) destaca entre sus contribuciones más relevantes:

1. Fortalecer la autoestima del alumnado.

2. Mejorar sustancialmente sus habilidades comunicativas.

3. Favorecer el desarrollo de las capacidades necesarias para diagnosticar situaciones, evaluar alternativas, ejecutar planes de acción y evaluar sus procesos y resultados.

4. Propiciar el desarrollo de actitudes pro-sociales y la capacidad de resistir y afrontar (resiliencia) las adversidades.

5. Promover el desarrollo de una conciencia ética y comprometida con el bien común.

6. Operar como un factor de protección que disminuye conductas de riesgo y de exclusión.

7. Desarrollar competencias básicas indispensables para la inserción en el mundo laboral, como la capacidad para la resolución de problemas prácticos y cotidianos, el cultivo de la creatividad y la iniciativa personal, el trabajo en equipo, la toma de decisiones y la capacidad de liderazgo.

8. Representar una ocasión para descubrir intereses y vocaciones relativas al futuro profesional.

A estos factores a tener en cuenta, sumamos los buenos resultados obtenidos en prácticas innovadoras de éxito previas en la aplicación de esta metodología en el Ciclo Formativo de Grado Superior de Integración Social de la misma familia profesional, y que vienen respaldadas por el reconocimiento a nivel nacional como buena práctica (Amigo y Galán, 2018, 2019; Solís, Lozano, Galán y Borrachero, 2019). Además, otras experiencias de la misma rama formativa como la llevada a cabo por Menéndez (2019) destacan desde la práctica reflexiva algunos de los beneficios importantes como: 1) su contribución a la formación de profesionales con autonomía y creatividad al flexibilizar los roles docente-discente, 2) el enriquecimiento mutuo y generación de conocimiento en el intercambio entidad educativa-entidad social y 3) la posibilidad de realización de prácticas guiadas, acompañadas y supervisadas en el propio proceso de enseñanza-aprendizaje más difícil de incorporar en los ciclos formativos de carácter social (Menéndez, 2019). Otros aspectos relevantes son los relacionados con su impacto en cuanto a la autoestima y motivación del alumnado. En este sentido, encontramos experiencias de FP, en las que se muestra el ApS como factor de mejora de la autoestima y, por tanto, de competencias personales y sociales en aquel tipo de alumnado que procede de historias de fracaso escolar (Millán y Aguilar, 2018). Otro aspecto de especial interés en la FP, debido a su orientación y capacitación para el empleo, es el relacionado con el descubrimiento de intereses y vocaciones en relación con el desempeño profesional (Martínez, 2014), factores que pueden contribuir a la motivación y la propia capacidad de autorregulación del aprendizaje. En este sentido, la motivación extrínseca para acceder a los estudios de FP está clara (la inserción laboral y la empleabilidad del alumnado), mientras que la intrínseca puede favorecerse a través de las estrategias promovidas por el profesorado (Maquilon y Hernández, 2011). El uso de la metodología de ApS contribuye a mejorar la motivación intrínseca, permitiendo la aplicación de estrategias de aprendizaje de distinto tipo, aunque destacan las socio-afectivas como parte imprescindible del compromiso social presente en las acciones de servicio comunitario orientada a la satisfacción de necesidades del entorno próximo. 
Por último, si se tienen en cuenta los factores de exclusión-inclusión educativa en España estudiados por Tarabini, Jacovkis y Montes (2017), el uso de ApS puede jugar un papel favorecedor de la inclusión educativa. Por un lado, favoreciendo la intervención frente los factores de exclusión en el aula como: falta de individualización del proceso de aprendizaje, falta de reconocimiento de los aspectos sociales que inciden en las actitudes escolares, falta de proximidad de los contenidos respecto a lo que se imparte en el aula y las expectativas personales (Tarabini et al., 2017). Por otro lado, el ApS puede ser una metodología afín a las recomendaciones de estos autores, ya que es una estrategia que permite reforzar la proximidad en la relación docente-alumno, así como contribuir a mejorar el conocimiento de las realidades socioculturales del alumnado evitando prácticas estigmatizantes o juicios y prestar atención a procesos emocionales que se dan en el aprendizaje. En este sentido, el ApS puede considerarse un instrumento al servicio de la educación inclusiva (Puig, Gijón, Martín y Rubio, 2011) y, en sí misma, una intervención educativa inclusiva (Martínez, 2014) por su utilidad para "desarrollar un conjunto de competencias básicas que solo pueden adquirirse a través de un trabajo global y contextualizado" (Puig et al., 2011, p. 45) y su "contribución a crear una sociedad más cohesionada y justa" (Martínez, 2014, p. 183).

Con todo lo expuesto, en el presente artículo se describe una experiencia desarrollada en el Ciclo Formativo de Grado Medio de Técnico de Atención a Personas en Situación de Dependencia (CFGM-TAPD), con el objetivo de contribuir a la mejora de la calidad de la FP y de las experiencias de aprendizaje del alumnado como futuros profesionales y ciudadanos-as comprometidos socialmente a través del ApS. Se trata de una propuesta educativa que pretende producir cambios individuales en las actitudes prosociales del alumnado, así como transformar las estructuras y dinamismos culturales que sustentan los estereotipos vinculados a la edad desde el futuro desempeño profesional. Es en este sentido que podemos definirla como una experiencia de ApS de tipo crítico (Chiva y Pallarès, 2018). Además de la descripción del proceso de implementación, se incluyen algunos resultados de la evaluación de la misma.

\section{CONTEXTO DE LA EXPERIENCIA}

El proyecto de innovación educativa en la FP Apd-intergeneracional se contextualiza en el CFGM-TAPD del IES Al-Qázeres (Cáceres, Extremadura), se inicia en el curso 2017-2018 y se consolida en del 2018/2019.

La capacitación del CFGM-TAPD se dirige a formar a los-as profesionales para ser capaces de: "atender a las personas en situación de dependencia, en el ámbito domiciliario e institucional, a fin de mantener y mejorar su calidad de vida, realizando actividades asistenciales, no sanitarias, psicosociales y de apoyo a la gestión doméstica, aplicando medidas y normas de prevención y seguridad y derivándolas a otros servicios cuando sea necesario (Real Decreto 1593/2011, de 4 de noviembre, artículo 4). De acuerdo con la Ley 39/2006 de Promoción de la Autonomía Personal y Social la dependencia se define como:

El estado de carácter permanente en que se encuentran las personas que, por razones derivadas de la edad, la enfermedad o la discapacidad, y ligadas a la falta o a la pérdida de autonomía física, mental, intelectual o sensorial, precisan de la atención de otra u otras personas o ayudas importantes para realizar actividades básicas de la vida diaria o, en el caso de las personas con discapacidad intelectual o enfermedad mental, de otros apoyos para su autonomía personal (Artículo 2).

Entre estos colectivos vulnerables se encuentra el configurado por las personas mayores. En nuestros días el envejecimiento de la población hace que vayan surgiendo nuevas necesidades sociales, entre ellas la de atender a una población envejecida en la que sigue 
creciendo en mayor medida la proporción de octogenarios; ya representan el 6,1\% de toda la población (Abellán, 2019) lo cual va demandando cada día perfiles profesionales cualificados y competentes.

En este sentido, se entiende que la formación directa con el colectivo es importante y, por tanto, eje de la FP. Motivo por el cual, el equipo educativo de profesionales del Departamento de SSC del IES Al-Qázeres vienen experimentando distintas metodologías activas para promover el desarrollo de las competencias profesionales, personales y sociales, indagando además modelos de evaluación formativa posibilitadores de la valoración de aprendizajes actitudinales y no sólo conceptuales y procedimentales, como se recoge en diversas publicaciones (Amigo y Galán, 2018, 2019; Solís et al., 2019).

En un diagnóstico inicial, realizado a través de la utilización de técnicas evaluativas de carácter cualitativo como entrevistas y grupos de debate, se detecta la presencia de actitudes y conductas estigmatizadas hacia el colectivo de intervención en el grupo de CFGM-TAPD en modalidad vespertina, (personas de entre 16 y 20 años, mayoritariamente). Se habla de personas mayores usuarias de centros de día o residencias de mayores. En concreto, personas que tienen una media de edad superior a 80 años que residen o pasan parte de su jornada diaria en estos centros y cuya realidad era desconocida para el alumnado debido a la distancia generacional. Desde la reflexión y análisis, el equipo docente determinó que la mejor manera de conocer al colectivo, así como sus necesidades y características, es a través del contacto directo durante el aprendizaje y no sólo limitado al periodo de prácticas. Por este motivo, se toma la decisión de promover el acercamiento intergeneracional y empatía como medio para contribuir al desarrollo integral, personal y profesional, de estas profesionales.

Para ello, en un primer momento se establece una alianza con el Centro de Día Francisco Chanclón, institución perteneciente a la Junta de Extremadura y que atiende a personas en situación de dependencia afectadas de Alzheimer y otras demencias. La regulación del servicio ofrecido por estos centros queda definida según la Ley 39/2006, de 14 de diciembre, de Promoción de la Autonomía Personal y Atención a las personas en situación de dependencia, desde la cual se determina que:

El servicio de Centro de Día o de Noche ofrece una atención integral durante el periodo diurno o nocturno a las personas en situación de dependencia, con el objetivo de mejorar o mantener el mejor nivel posible de autonomía personal y apoyar a las familias o cuidadores. En particular, cubre, desde un enfoque biopsicosocial, las necesidades de asesoramiento, prevención, rehabilitación, orientación para la promoción de la autonomía, habilitación o atención asistencial y personal (artículo 24).

La participación activa del equipo de profesionales del Centro Día con el equipo educativo del CFGM-TAPD propició que se estableciera una alianza formalizada mediante un Proyecto Intergeneracional (PI) de colaboración entre el IES Al-Qázeres y el Centro de Día Francisco Chanclón a principios del curso 2017/2018, siguiendo los protocolos de actuación de los PI coordinados desde la Consejería de Sanidad y Políticas Sociales, a través del Servicio Extremeño de Promoción de la Autonomía y Atención a la Dependencia (SEPAD) (https://saludextremadura.ses.es/sepad/programas-intergeneracionales), y la Consejería de Educación y Empleo de la Junta de Extremadura (https://www.educarex.es/atenciondiversidad/intergeneracionales.html).

Los PI son"...actividades o programas que incrementan la cooperación, la interacción y el intercambio entre personas de diferentes generaciones. Implican compartir habilidades, conocimientos y experiencias entre jóvenes y mayores" (Ventura-Merkel y Lidoff, 1983; citado en 
Newman y Sánchez, 2007:38). Con este tipo de proyectos se consigue desarrollar las competencias personales y sociales propias que este perfil profesional debe adquirir para el correcto desempeño de su profesión en el futuro, como es una actitud respetuosa con el colectivo formado por las personas mayores y sus necesidades. De esta forma, conseguir "aprovechar de modo beneficioso el potencial de la intergeneracionalidad en tanto encuentro e intercambio entre personas y grupos pertenecientes a distintas generaciones" (Newman y Sánchez, 2007, p. 38).

Estos motivos justifican que el PI fuese la herramienta utilizada para atender la necesidad social de disminuir o combatir los edadismos, es decir, el "mantenimiento de estereotipos o actitudes prejuiciosas hacia una persona, únicamente por el hecho de ser mayor" (Bárcena, Iglesias, Galán y Abella, 2009:47). En el curso siguiente, 2018/2019, fruto de los resultados del primer curso, se establecieron nuevas alianzas entre entidades sociales, en esta ocasión con la Residencia de Mayores Cervantes con la que se formalizó un nuevo PI. En esta ocasión, debido a la cercanía del establecimiento residencial al Centro Escolar, se definieron actividades de temporalización semanal que fueron enmarcadas en un módulo concreto del currículum del CFGM-TAPD. La docencia de dicho módulo se consideró adecuada para el desarrollo del PI a través de la metodología de ApS, debido a su contenido teórico-práctico y carácter aplicado, así como por su duración y carga lectiva.

Es por ésto que se describen dos líneas de intervención, una con el Centro de Día Francisco Chanclón y otra con la Residencia Cervantes. La primera línea de actuación AlQázeres-Francisco Chanclón, denominada "Edadismo-no", consistente en la celebración de encuentros entre personas jóvenes y mayores en los que pudieran compartir momentos de su realidad cotidiana. La segunda línea de actuación Al-Qazeres-Cervantes, denominada "Dinamización del espacio de la memoria", se ejecutaba y dinamizaba con una atención individualizada, asignando a cada persona mayor un-a compañero-a de actividad, en conexión con los contenidos curriculares y las competencias transversales trabajadas en el Centro a partir de las celebraciones pedagógicas.

En conclusión, se parte de una NECESIDAD (Mejorar las competencias profesionales y personales de las personas jóvenes en formación que van a desarrollar su desempeño profesional con personas mayores), se define un SERVICIO que sirva para satisfacer dicha necesidad (Crear espacios de encuentro entre generaciones), para lo que se requerirá adquirir unos APRENDIZAJES (Competencias del currículo del CFGM-TAPD). Para aprender haciendo y prestando el servicio, se establecen ALIANZAS con dos entidades sociales en un intercambio recíproco. Además, la experiencia de ApS se contextualiza en el marco de los Objetivos de Desarrollo Sostenible (ODS). Todos ellos ingredientes básicos para un proyecto de ApS (Batlle, 2013, 2018).

\section{Objetivos}

Los aprendizajes curriculares se vinculan al conjunto de competencias profesionales y personales que vienen recogidas en el Real Decreto1593/2011, de 4 de noviembre, por el que se establece el Título de TAPD en su artículo 4. Para el logro de los objetivos de aprendizaje y competencias se define como metodología paraguas el ApS. Se señalan a continuación los objetivos educativos, de servicio y los propósitos docentes en relación a la propuesta:

\section{A. Como objetivos educativos de este proyecto se marcan los siguientes para las personas que participan del centro educativo:}

1. Desarrollar una imagen positiva y libre de prejuicios y no estereotipada de las personas mayores. 
2. Adquirir competencias profesionales y favorecedoras de la empleabilidad del alumnado propias del Título.

3. Desarrollar valores relacionados con el sentido cívico y de la responsabilidad hacia la comunidad.

4. Asunción de la propia identidad profesional y personal conforme al código ético de la profesión.

\section{B. Como objetivos del servicio se marcan los siguientes objetivos:}

1. Disminuir las consecuencias que tienen el aislamiento y la soledad a la que se enfrentan algunas personas mayores en nuestra sociedad.

2. Facilitar espacios de encuentro entre personas jóvenes y mayores que fomenten el intercambio de comunicación y creando redes de apoyo entre las mismas.

3. Combatir la imagen estereotipada que existe entre las generaciones de personas jóvenes y personas mayores.

4. Mejorar la autoestima y el bienestar emocional de las personas mayores.

5. Desarrollar competencias digitales para un manejo básico de las tecnologías entre las personas destinatarias del servicio.

\section{Propósitos de la propuesta y del equipo educativo:}

1. Propiciar experiencias de metodologías innovadoras y activas como el ApS.

2. Potenciar el conocimiento de la heterogeneidad de las personas mayores y promover el desarrollo de actitudes de respeto hacia la persona humana.

3. Mejorar las competencias sociales y personales en el entorno educativo y laboral.

4. Fomentar la competencia de "Aprender a Aprender".

5. Mejorar la valoración del colectivo de las personas mayores y la importancia de su función en la sociedad entre las personas jóvenes.

Por último, este proyecto y sus objetivos de aprendizaje y servicio se vinculan directamente con el ODS 1. Promover sociedades pacíficas e inclusivas para el desarrollo sostenible, facilitar acceso a la justicia para todos y crear instituciones eficaces, responsables e inclusivas a todos los niveles. Dicho objetivo se vincula directamente con las competencias de nuestro perfil profesional, ya que consideramos que en la medida en que la sociedad incluya a todas las personas que la conforman sin discriminación por edad conseguiremos que sean pacíficas e inclusivas. Además, desde esta experiencia se trabajan el ODS 5 Lograr la igualdad entre los géneros empoderar a todas las mujeres y niñas, y el ODS 4 Garantizar una educación inclusiva, equitativa y de calidad y promover oportunidades de aprendizaje durante toda la vida para todos (UNESCO, 2016, 2017).

\section{Participantes}

Alumnado del CFGM-TAPD. Estamos hablando de dos grupos de alumnas-os. El grupo del primer curso estaba formado por 25 personas de entre 16 y 20 años, mujeres salvo un hombre. El segundo curso estaba formado por 23 mujeres de entre 17 y 20 años. Las características que presentaban los dos grupos eran similares. Procedían de entornos rurales en su mayoría, municipios en los que no había posibilidad de cursar CFGM de esta familia profesional; otras procedían del medio urbano, la mayoría de otros centros educativos o centros de educación de adultos; muy pocas personas procedían del propio IES. El nivel cognitivo del alumnado era medio-bajo, presentando serias dificultades de aprendizaje en algunos casos. La gran mayoría había repetido algún curso o habían obtenido la titulación de la ESO cursando programas especiales como PRAGE O PMAR. Otras personas habían abandonado el sistema educativo y habían accedido a través de pruebas acceso. Las menos procedían de programas de FP Básica. Las expectativas principales con respecto a las enseñanzas eran conseguir una 
capacitación para acceder al mundo laboral o bien utilizar las enseñanzas de CFGM para acceder a un ciclo de Grado Superior de la misma familia profesional.

Personas usuarias del Centro de Día. Eran una veintena de personas mayores de 85 años en su mayoría, mujeres salvo tres hombres, que presentaban demencia relacionada con el Alzheimer. Estas personas asisten al Centro de Día parte de la jornada, pero residen en sus hogares con un nivel de dependencia grave. El nivel cultural de estas personas era medio, aunque su nivel de motivación y participación eran muy altos.

Personas usuarias de la Residencia Cervantes. Eran 20 mujeres y 2 hombres, la mayoría con más de 90 años, incluso había una centenaria, con un nivel de dependencia grave, poca movilidad y diversas patologías asociadas a la edad. El nivel cultural de estas personas era medio-bajo (algunas apenas sabían leer). El nivel de motivación era alto y se mostraban participativas.

\section{DESARROLLO DE LA EXPERIENCIA}

El desarrollar este doble proyecto, posibilita enlazar dos líneas de actuación que dan una visión global al alumnado de su futuro profesional.

Por un lado, y como primera línea de actuación, Al-Qázeres-Francisco Chanclón, con su área de intervención denominada "Edadismo-no". En esta línea la realización de las actividades programadas proporcionan una visión de la tarea asistencial no sanitaria y psicosocial que llevan a cabo los y las TAPD en el centro de trabajo, en este caso un Centro de Día. El alumnado se convierte en un-a trabajador-a más del centro, acudiendo en pequeño grupo para integrarse en el quehacer diario participando en actividades propias del desempeño laboral (Tabla 1). En este caso, se favorece la formación práctica, similar a la FP Dual, aplicando los conocimientos adquiridos en el aula en un entorno laboral formal, aprendiendo a actuar ante las posibles contingencias y siendo guiados por las-los profesionales de la institución. En este tipo de actividades se produce además un intercambio informal de experiencias y aprendizajes recíprocos entre las personas usuarias y nuestro alumnado, enriqueciendo más aún su formación y adquisición de destrezas y actitudes. Del mismo modo, y con el agrupamiento de gran grupo se llevan a cabo actividades puntuales con carácter lúdico recreativas. En el Centro de Día Francisco Chanclón, se realizan tres actividades (una por trimestre) en las que se acerca a las personas mayores al Centro Educativo, cambiando su escenario habitual por el Instituto para el desarrollo de las actividades.

En esta línea de actuación participó el alumnado de $1^{\circ}$ curso del CFGM-TAPD durante el curso 2017/2018, y en el actual curso académicos, en las actividades grupales todo el alumnado del ciclo, tanto $1^{\circ}$ como $2^{\circ} \mathrm{CFGM}$, hasta el mes de marzo, momento en el que el segundo curso comience su periodo de Formación en Centros de Trabajo, continuando con las actividades en pequeño grupo únicamente el alumnado de primer curso.

La segunda línea de actuación, Al-Qázeres-Cervantes, es la que se lleva a cabo en el Centro Residencial "Cervantes" de Cáceres. Esta línea consiste en la realización de un programa de actividades denominadas "Dinamización del espacio de la memoria" con el objetivo que el alumnado de TAPD ponga en práctica los contenidos teóricos que aprende en el aula, dándole sentido a sus aprendizajes. Además, las personas mayores también participan en las actividades que se llevan a cabo en el Instituto (celebraciones pedagógicas). En el encuentro entre jóvenes y mayores suman más los beneficios emocionales y personales que los meramente académicos y residenciales. De forma que se consigue mejorar la calidad de vida y el bienestar de las personas mayores a la par que desarrollar empatía y el conocimiento no estereotipado del 
colectivo por parte de las-os jóvenes en formación. Dichas actividades se llevan a cabo en gran grupo, permitiendo, al igual que la primera línea de actuación el intercambio espontáneo de vivencias $y$, por ende, de bagaje formativo.

A continuación, describimos las distintas fases de la secuencia:

\section{Etapa de preparación y análisis de la realidad:}

Como se indica en epígrafes anteriores, se parte del análisis previo de necesidades y de la utilización de técnicas de Diagnóstico Social Participativo (DSP) con la participación dialógica del alumnado en el análisis crítico de la realidad del contexto comunitario -desde el diagnóstico y análisis hasta la priorización de necesidades- favorece el compromiso del alumnado en el desarrollo, transformación y mejora del entorno (Traver, Sales y Moliner, 2018). En este sentido, estas técnicas son empleadas como herramienta de transformación social e instrumento de concienciación que facilita la toma de conciencia crítica y el empoderamiento ciudadano (Aguirre et al., 2017, Freire, 1974; citado en Traver et al., 2018). Las fases de detección, análisis y priorización de las necesidades vienen caracterizadas por el análisis crítico de la realidad a través de la observación.

\section{Etapa de diseño y planificación de las actividades:}

Partiendo del conocimiento de la realidad, se diseñan y la planificación cada una de las líneas de intervención.

En la primera línea, por el equipo educativo, con la participación del alumnado en la toma de decisiones y aspectos organizativos como:

- Preparación de encuentros. Técnicas de debate y reflexión ¿qué queremos hacer?

- Investigación y análisis de las propuestas.

- Diseño, planificación preparación de las actividades en grupos de trabajo por parte de las-os jóvenes.

En la segunda línea de actuación, se desarrolló una planificación de un módulo en concreto, que se adaptó a la programación diseñada en ese curso. La organización de "Dinamización del espacio de la memoria" se realizaba así:

- Organización curricular del módulo en bloques de contenido y temas. Se procedía a la realización de actividades de aprendizaje de cada tema. Partiendo de esa base se pasaba al trabajo práctico de investigación-acción.

- Elaboración de secuencias de actividades de una sesión de duración en grupos de trabajo rotatorios por bloques de contenido y adaptadas a las necesidades observadas en el grupo de personas mayores.

- Elaboración de recursos audiovisuales y en otros formatos (videos, fichas, recortables...) adaptados a los y las destinatarias, para lo que se analizaron y exploraron aplicaciones digitales y de software libre.

- Búsqueda de recursos comunitarios (por ejemplo: la colaboración de un grupo de teatro).

\section{Etapa de implementación del servicio y seguimiento de los aprendizajes:}

Como hemos explicado el proyecto se articula en dos partes por un lado las intervenciones que se hacen en el Centro de Día Francisco Chanclón y por otro las que se hacen en la Residencia Cervantes (Tabla 1). Como indicamos anteriormente, las actividades de aprendizaje y de reflexión estaban basadas en las técnicas de DSP puesto que iniciaban procesos de concienciación de las personas que participaban al tomar conciencia crítica de la propia realidad con el ánimo de emprender acciones justas para su transformación y mejora (Traver et al., 2018). Después de cada una de las actividades descritas en la etapa de implementación se procedía a 
una sesión de reflexión que consistía en la realización de técnicas de grupo en las que se compartía a través de la expresión de emociones cómo había sido el desarrollo de la actividad en la que habían sido protagonistas, las dificultades y facilidades que habían encontrado así como las posibles mejoras de cara a la siguiente actividad.

El resultado de estas técnicas de grupo se recogía en los diarios reflexivos de campo y servían de instrumento de evaluación (Moon, 2004). De esta forma se conseguía que las-os participantes se posicionan como dueñas-os de su propia información, pudiendo realizar una reflexión crítica sobre la imagen construida con la información recogida con anterioridad a través de la observación de la realidad (Traver et al., 2018, p. 27).

Tabla 1. Secuenciación de actividades de las líneas de actuación del proyecto Apd Intergeneracional.

\begin{tabular}{|c|c|c|c|}
\hline $\begin{array}{l}\text { Secuencia de } \\
\text { actividades }\end{array}$ & $\begin{array}{c}\text { LÍNEA DE } \\
\text { ACTUACIÓN } \\
\text { FRANCISCO } \\
\text { CHANCLÓN } \\
\text { EMERGENTE (sólo en } \\
\text { 2017-2018) }\end{array}$ & $\begin{array}{c}\text { LÍNEA DE } \\
\text { ACTUACIÓN } \\
\text { FRANCISCO } \\
\text { CHANCLÓN } \\
\text { CONSOLIDADA } \\
\text { (2018-2019) }\end{array}$ & $\begin{array}{c}\text { SEGUNDA LÍNEA DE } \\
\text { ACTUACIÓN “DINAMIZACIÓN } \\
\text { ESPACIO DE LA MEMORIA } \\
\text { CERVANTES” } \\
\text { (2018/2019) }\end{array}$ \\
\hline Iniciales & $\begin{array}{l}\text { Taller de formación y } \\
\text { sensibilización para el } \\
\text { profesorado y el } \\
\text { alumnado. } \\
\text { Diseño del blog que } \\
\text { recogerá la experiencia } \\
\text { "edadismo-no". }\end{array}$ & $\begin{array}{l}\text { Taller de sensibilización } \\
\text { y formación entre } \\
\text { iguales. }\end{array}$ & $\begin{array}{l}\text { Actividades de conocimiento. } \\
\text { Análisis de las necesidades de las } \\
\text { personas usuarias y planificación } \\
\text { de las actividades a realizar } \\
\text { relacionadas con la Atención y el } \\
\text { Apoyo Psicosocial. }\end{array}$ \\
\hline De Desarrollo & $\begin{array}{l}\text { Taller de } \\
\text { gerontogimnasia. } \\
\text { Taller de atención } \\
\text { sanitaria no asistencial. } \\
\text { Taller de rehabilitación } \\
\text { cognitiva. }\end{array}$ & $\begin{array}{l}\text { Aprendiendo a ser } \\
\text { TAPD: Un día en el } \\
\text { Centro de Día (1 vez } \\
\text { por trimestre). } \\
\text { Espacios de encuentro } \\
\text { cultural } \\
\text { intergeneracional } \\
\text { - Navidad extremeña } \\
\text { - Carnaval cacereño el } \\
\text { pelele } \\
\text { - Un día en el IES Al- } \\
\text { Qázeres }\end{array}$ & $\begin{array}{l}\text { ACTIVIDADES CONTINUADAS } \\
\text { EN EL TIEMPO: } \\
\text { Diseño y planificación de los } \\
\text { talleres por parte del alumnado } \\
\text { con la supervisión docente. } \\
\text { Realización de las actividades } \\
\text { planificadas en la residencia. } \\
\text { Elaboración de un porfolio en el } \\
\text { que se recoge toda la información. } \\
\text { ACTIVIDADES PUNTUALES* } \\
\text { Surgen de la iniciativa del } \\
\text { alumnado y suponen una } \\
\text { participación activa de la entidad } \\
\text { social en el centro educativo. } \\
\text { - Gala Cervantes. } \\
\text { - Radio Onda Al -Qázeres. } \\
\text { - Día } 25 \text { N "Puerta Violeta". } \\
\text { - Día de la Mujer "Referentes". } \\
\text { - Concurso micro relatos Día del } \\
\text { Centro. } \\
\text { - Teatro Intergeneracional "Las } \\
\text { violeteras". }\end{array}$ \\
\hline Finales & $\begin{array}{l}\text { Jornadas de } \\
\text { Convivencia. } \\
\text { Evaluación y Reflexión. }\end{array}$ & $\begin{array}{l}\text { Celebración fin de } \\
\text { curso. } \\
\text { Evaluación y reflexión. }\end{array}$ & $\begin{array}{l}\text { Jornada de celebración y clausura } \\
\text { Evaluación y reflexión. }\end{array}$ \\
\hline
\end{tabular}

Fuente: Elaboración propia.

\section{Etapa de difusión:}

Los canales que utilizamos para la difusión del proyecto fueron los paneles informativos del centro educativo (cartelería y publicidad), radio y las redes sociales institucionales (blogs https://edadismo-no.blogspot.com/ y https://apdintergeneracional.blogspot.com/, Facebook: sscalqazeres, Twitter: @sscalqazeres, Instagram @sscalqazeres). Además de eso se procedió 
a la difusión del proyecto a través de los canales de comunicación de las entidades sociales y, en concreto, del SEPAD en la red de PI.

El proyecto también ha sido difundido con la comunidad profesional socio-educativa a través de actividades de formación inicial y continua promovidas del grupo promotor de ApS de Extremadura para difundir la metodología y dar a conocer las experiencias regionales (Solís et al., 2019; Solís y Mayor, en prensa).

\section{Reflexión y evaluación:}

Los principales instrumentos de evaluación empleados fueron el diario reflexivo individual y el portfolio, utilizando como medio el blog permitiendo así seguir ciclos de acción, reflexión y toma de decisiones. Siendo éstos instrumentos, reflexivos y basados en el desempeño, los más idóneos para llevar a cabo una evaluación formativa que permita empoderar al alumnado a través de la autoconsciencia de sus propios procesos de aprendizaje y la identificación de sus posibilidades de mejora (UNESCO, 2017). Es por ello que la autoevaluación y la coevaluación se consideran métodos imprescindibles y que permiten al alumnado reflexionar sobre aspectos como "la transformación personal, la comprensión más holística de la investigación crítica, y el compromiso y la participación cívica" (UNESCO, 2017, p. 57).

En cuanto a las personas receptoras del servicio, se llevaron a cabo entrevistas personalizadas para valorar su grado de satisfacción, registradas a través de grabaciones de audio que, se difunden a través de la radio educativa del centro. Las entrevistas medían indicadores tales como el grado de participación, el nivel de motivación, el nivel de mejora de la calidad emocional y bienestar psicológica, entre otros.

En relación al equipo educativo y el equipo colaborador de las entidades sociales se utilizaron rúbricas de evaluación para evaluar el diseño, planificación e implementación de las actividades, en ellas se tuvieron en cuenta los siguientes indicadores: impacto en la implementación de estas metodologías activas en la formación del alumnado, repercusión de los aprendizajes procedimentales en el aula, mejora del clima grupal, motivación del alumnado, mejora del rendimiento académico y escolar. Con el uso de estos instrumentos se conseguía la reflexión y mejora de la práctica docente lo que determinó la consolidación del proyecto.

\section{RESULTADOS}

El análisis y evaluación de la experiencia se ha llevado a cabo mediante la aplicación de la Rúbrica para la autoevaluación de proyectos de aprendizaje servicio (GREM, 2014; Rubio, Puig, Martín, y Palos, 2015; Puig, Martín y Rubio, 2017; Martín, Puig, Palos y Rubio, 2018). En línea con otras experiencias de evaluación formativa y colaborativa, la rúbrica se ha adaptado en versión electrónica y aplicado a través de la aplicación web Corubrics de Google Drive (Cebrián, 2018; Romero y Torrebadella, 2018).

La elección de este soporte para la elaboración de e-rúbricas, se ha basado en las posibilidades que ofrece para trabajar de manera colaborativa online tanto con el alumnado como entre el equipo docente (Tomero y Torrebadella, 2018). De esta forma, resulta una herramienta útil y aplicable a diferentes modalidades de evaluación formativa (evaluación $360^{\circ}$, autoevaluación, evaluación entre pares, evaluación de equipos, evaluación de productos, evaluación ipsativa, etc) (Cebrián, 2018, p. 67).

A continuación, se presentan los resultados de la aplicación de la rúbrica a la autoevaluación del proyecto Apd Intergeneracional (Tabla 2 y Gráfico 1). 
Tabla 2. Resultados de la aplicación de la rúbrica de autoevaluación al proyecto Apd Intergeneracional.

\begin{tabular}{|c|c|c|c|c|}
\hline Dinamismos/ Niveles & $\mathbf{I}$ & II & III & IV \\
\hline Necesidades & Ignoradas & Presentadas & Decididas & Descubiertas \\
\hline Servicio & Simple & Continuado & Complejo & Creativo \\
\hline Sentido del servicio & Tangencial & Necesario & Cívico & Transformador \\
\hline Aprendizaje & Espontáneo & Planificado & Útil & Innovador \\
\hline Participación & Cerrada & Delimitada & Compartida & Liderada \\
\hline Trabajo en grupo & Indeterminado & Colaborativo & Cooperativo & Expansivo \\
\hline Reflexión & Difusa & Puntual & Continua & Productiva \\
\hline Reconocimiento & Casual & Intencional & Recíproco & Público \\
\hline Evaluación & Informal & Intuitiva & Competencial & Conjunta \\
\hline Partenariado & Unilateral & Dirigido & Pactado & Integrado \\
\hline $\begin{array}{l}\text { Consolidación } \\
\text { Centros }\end{array}$ & Incipiente & Aceptada & Integrada & Identitaria \\
\hline $\begin{array}{l}\text { Consolidación } \\
\text { Entidades }\end{array}$ & Incipiente & Aceptada & Integrada & Identitaria \\
\hline
\end{tabular}

Fuente: Elaboración propia.

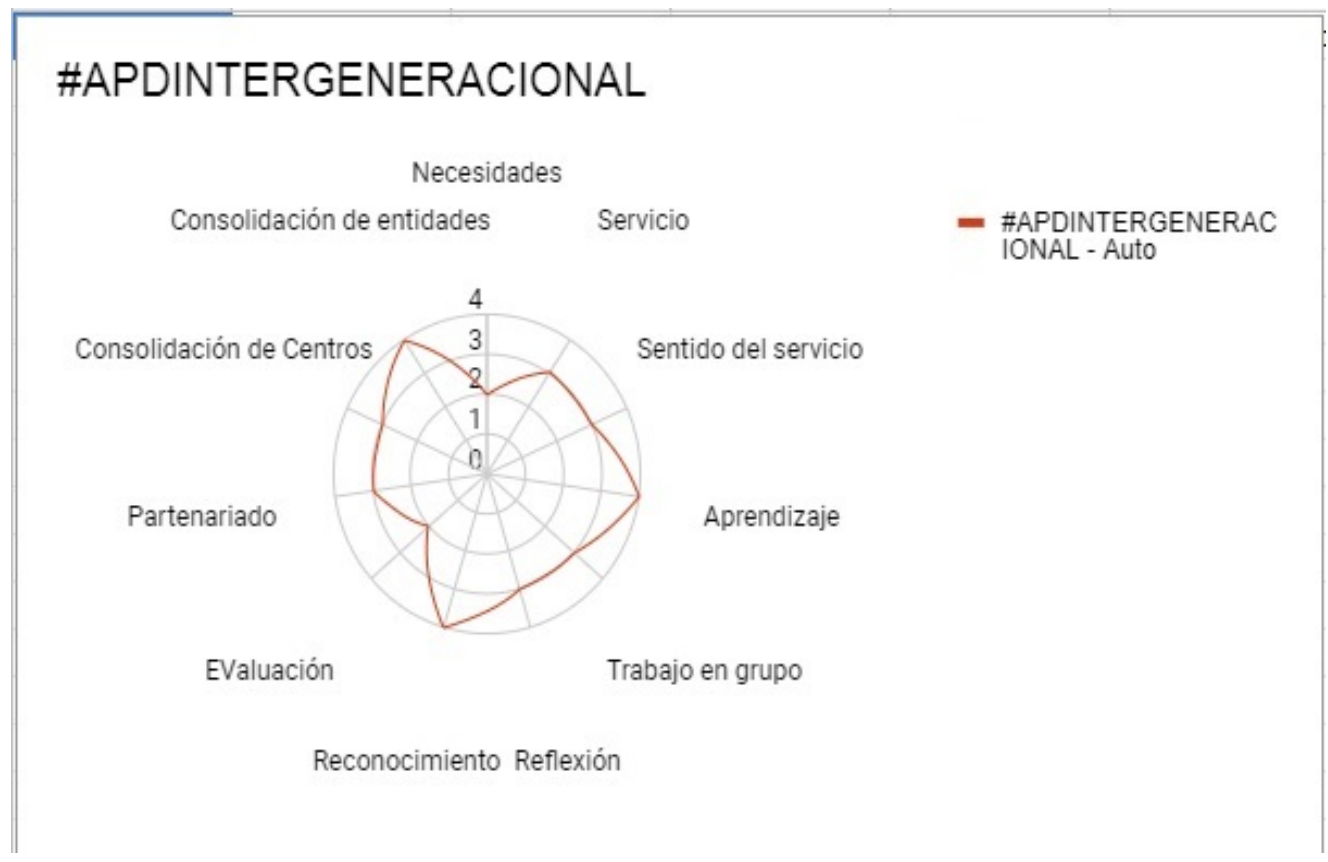

Figura 1. Gráfico de araña de la evaluación de la experiencia Apd Intergeneracional. Fuente: Elaboración propia.

En el análisis de los datos de la rúbrica se identifican como puntos fuertes, la consolidación de las entidades, el reconocimiento que el proyecto de ApS genera en el resto de la comunidad y el aprendizaje de las competencias profesionales, personales y sociales. Se destaca a menor escala indicadores como el sentido del servicio el servicio, el trabajo en grupo y el partenario creado. Como puntos más débiles se destaca principalmente la dimensión relacionada con la necesidad social, explicándose por el hecho de que en un primer momento no es sentida por el alumnado, sino que es percibida por el equipo docente (Pérez-Serrano, 2003). 
Matizamos algunos de los aspectos en relación a las dimensiones de la rúbrica, enriqueciendo los resultados con los obtenidos a través del análisis documental de las producciones del alumnado e informes recopilados en el transcurso de la experiencia.

En relación a la valoración de los aprendizajes del alumnado:

1. El uso de la metodología de ApS muestra ser útil para mejorar el rendimiento escolar, aumentar la motivación y la comunicación del grupo de alumnas. La aplicación de esta experiencia ha hecho que durante estos dos cursos descendiera el nivel de absentismo escolar en el alumnado, así como mejorado los resultados académicos y de promoción. Como dato que se recoge en las memorias del IES, decir que de la promoción 2016-2017 sólo promocionan a segundo curso 13 personas de las 25 matriculadas, mientras que en los cursos en los que se aplica la experiencia, promocionaron la totalidad del grupo.

2. El desarrollo de estas experiencias intergeneracionales consiguen que las personas que se matriculan en estas enseñanzas cambien su mirada con respecto al colectivo de personas mayores y a las necesidades de comunicación, estima y afecto que presentan. Se mejora el desarrollo de valores relacionados con el sentido cívico y la responsabilidad hacia las personas mayores

Si tenemos en cuenta las dimensiones que se refieren al partenariado a la consolidación del proyecto de ApS tanto en el centro como en la entidad social. Los resultados son los siguientes:

1. Las personas mayores se sienten más acompañadas por el grupo-clase, y además manifiestan verbalmente ese bienestar. Los espacios de encuentro favorecen la comunicación, la creación de redes, es descubrimiento de habilidades personales, de tradiciones, de cultura entre unas generaciones y otras.

2. Se fidelizan las relaciones entre los centros educativos y las entidades sociales que trabajan en la comunidad en la que se inserta el proyecto, favoreciendo futuras colaboraciones.

\section{CONCLUSIONES}

La metodología de ApS en esta ocasión ha conseguido que los-as jóvenes aprendan haciendo tareas propias de la profesión. De esta forma coincidimos con Saz y Ramos (2015) al considerar que el ApS puede "lograr mayor formación profesional y personal por parte de nuestros estudiantes". Además, desde la FP, puede contribuir a revalorizar y cambiar la imagen social deteriorada de la FP en nuestro país (Echevarría y Martínez, 2018). En definitiva, apostamos por una FP de calidad (Echevarría y Martínez, 2019), "útil, productiva, responsable y sostenible en nuestro contexto" (Marhuenda, Chisvert y Palomares, 2016, p. 60), que sea también transformadora, promotora de conocimiento y compromiso social.

Otro de los logros a destacar en este caso concreto, es el alto grado de motivación de losas jóvenes hacia la investigación y la reflexión sobre diferentes estrategias de atención a las personas mayores para mejorar su calidad de vida. Así, podemos señalar como algunos estudios como el llevado a cabo por Santos-González y Sarceda-Groso (2017) han resaltado la buena motivación del alumnado de FP hacia las prácticas en entornos profesionales. Sin embargo, también destacan la necesidad de promover el conocimiento de dichos espacios laborales con anterioridad a su incorporación. A este respecto, experiencias de ApS a lo largo de la FP como 
la aquí descrita pueden contribuir a acercar al alumnado a entornos y experiencias prácticas reales, respondiendo a sus expectativas y mejorando sus motivaciones previas.

La experiencia va creciendo en función de la implicación de las personas participantes, unas personas contagian a otras y así, en el curso 2019/2020, el proyecto sigue consolidado y replicándose. Los-as participantes son las que hacen que la metodología de ApS dé significado a los aprendizajes y, a la vez, que los servicios respondan a necesidades percibidas o sentidas. Ellas y ellos son las que animan y alientan a las entidades sociales a implicarse en el desarrollo de estas experiencias. En este sentido, como en los proyecto de ApS en cascada, las-os futurasos profesionales "se convierten, a través de un proyecto de ApS, en promotores de ApS" (García y Cotrina, 2015, p. 18), en este caso, en las entidades sociales.

Por último, subrayar que el éxito de la experiencia es debido en gran parte por el empeño de las-os profesionales de la entidad social, quienes ponen a disposición del equipo educativo y del alumnado todos los medios para que ésto se materialice. Finalmente, señalar que este proyecto, así como sus resultados, ha sido germen e inspiración de otros proyectos en distintas familias profesionales del IES Al-Qázeres y distintos niveles educativos apoyándose en la filosofía del ApS. Es prueba de, lo que en palabras de Roser Batlle (2013), podemos calificar de "contagio de una revolución pedagógica necesaria".

\section{BIBLIOGRAFÍA}

Abellán, A. (Dir.) (2019). Un perfil de las personas mayores en España, 2019. Indicadores estadísticos básicos. Madrid: Informes Envejecimiento en red $\mathrm{n}^{0}$ 22. Recuperado de http://envejecimiento.csic.es/documentos/documentos/enred-indicadoresbasicos2019.pdf

Amigo, A. y Galán, M.J. (2018). Experiencias de FP. IES Al-Qázeres. En A. Saiz, P. Debén, M. de Quinto y V. Rivera (Coords.). IX Premio Nacional de Educación para El Desarrollo "Vicente Ferrer" (pp 150163). Madrid: AECID.

Amigo, A. y Galán, M.J. (2019). El viaje de las personas refugiadas: del conocimiento a la solidaridad. CONVIVES, 25, 54-61. Recuperado de https://convivesenlaescuela.blogspot.com/2019/03/frente-aldiscurso-del-odio-convivencia.html

Bárcena, C., Iglesias, J. A., Galán, M. I., y Abella, V. (2009). Dependencia y edadismo. Implicaciones para el cuidado. Revista Enfermería CyL, 1(1), 46-52. Recuperado de http://www.revistaenfermeriacyl.com/index.php/revistaenfermeriacyl/article/view/18

Batlle, R. (2013). El Aprendizaje-Servicio en España: el contagio de una revolución pedagógica necesaria. Madrid: PPC.

Batlle, R. (2018). Guía práctica de Aprendizaje-servicio. Barcelona: Santillana.

Castel, R. (1997). Metamorfosis de la cuestión social. Una crónica del salariado. Buenos Aires: Paidós.

Cebrián, M. (2018). Modelo de evaluación colaborativa de los aprendizajes en el prácticum mediante Corubric. Revista Prácticum, 3(1), 62-79. Recuperado de https://revistapracticum.com/index.php/iop/article/view/44

Chiva, O. y Pallarès, M. (2018). Aprendizaje-servicio: pasaporte para un futuro mejor. En O. Chiva y M. Pallarès (Coords.), Aprendizaje-servicio: pasaporte para un futuro mejor (pp. 9-17). Sevilla: Ediciones Egregius.

Echevarría, B. y Martínez, P. (2019). La investigación sobre Formación Profesional Inicial en España (20052017). Cuadernos de pedagogía, (499), 65-69.

Echeverría, B. y Martínez, P. (2018). Estrategias de mejora en la implantación de la Formación Profesional Dual en España. EKONOMIAZ, 94(02), 178-203. Recuperado de https://ideas.repec.org/a/ekz/ekonoz/2018211.html

Flores, M., Ortega, M. y Vallejo, S. (2019). Experiencias inclusivas intergeneracionales: ¿un nuevo horizonte para la inclusión de personas mayores y menores?. Miscelánea Comillas, 77(150), 139152. Recuperado de https://fund-encuentro.org/index.php/miscelaneacomillas/article/view/11716

FUNDACIÓN FOESSA (2019). VIII informe sobre Exclusión y desarrollo social en España 2019. Madrid: Fundación FOESSA y Cáritas Española.

García, M. y Cotrina, M. J. (2015). El aprendizaje y servicio en la formación inicial del profesorado: de las prácticas educativas críticas a la institucionalización curricular. Profesorado: Revista de Currículum y Formación del Profesorado, 19(1), 8-25. Recuperado de http://www.ugr.es/local/recfpro/rev191ART1.pdf 
GREM (2014). Rúbrica para la autoevaluación y la mejora de los proyectos de Aprendizaje-Servicio. Barcelona: Fundación Jaume Bofill. Recuperado de http://bit.do/eSTbb

Ley 39/2006, de 14 de diciembre, de Promoción de la Autonomía Personal y Atención a las personas en situación de dependencia. BOE, núm. 299, de 15 de diciembre de 2006.

Ley Orgánica 8/2013, de 9 de diciembre, para la mejora de la calidad educativa (LOMCE). BOE, núm. 295, de 10 de diciembre de 2013.

Maquilón, J.J. y Hernández, F. (2011). Influencia de la motivación en el rendimiento académico de los estudiantes de formación profesional. REIFOP, 14(1), 81-100. Recuperado de http://redined.mecd.gob.es/xmlui/handle/11162/81386

Marhuenda, F., Chisvert, M.J. y Palomares-Montero, D. (2016). La formación profesional dual en España. Consideraciones sobre los centros que la implementan. RIO,(17), 43-63. DOI: https://doi.org/10.17345/rio17.43-63

Martín, Á. (2015). Contextualización de los centros de formación profesional en su entorno: Retos y oportunidades en la sociedad del conocimiento (Tesis doctoral). Sevilla: Universidad de Sevilla.

Martín, X., Puig, J. M., Palos, J., \& Rubio, L. (2018). Enhancing the quality of Service-Learning practices. Enseñanza \& Teaching, 36(1), 111-128. DOI: https://doi.org/10.14201/et2018361111128.

Martínez, B. (2014). Aprendizaje-servicio y educación inclusiva. Educación y futuro, (30), 183-208. Recuperado de https://dialnet.unirioja.es/servlet/articulo?codigo $=4685097$

Mateos, S., Cerrillo, R., \& Tello, I. (2018). Aprendizaje-servicio en formación profesional. En Avances en democracia y liderazgo distribuido en educación: Actas del II Congreso internacional de liderazgo y mejora de la educación (pp. 285-288). Red de Investigación sobre Liderazgo y Mejora de la Educación (RILME).

Menéndez, M.C. (2019). Cambiando los roles educativos: una experiencia de aprendizaje-servicio en la Formación Profesional de Grado Superior. Cuadernos de pedagogía, (496), 55-61.

Millán, A. y Aguilar, J.M. (2018). Impacto y beneficio del Aprendizaje-Servicio en la autoestima del alumnado de formación profesional básica de Informática. En O. Chiva y M. Pallarés (Coords.), AprendizajeServicio: pasaporte para un futuro mejor (pp. 95-104). Sevilla: Ediciones Egregius.

Moon, J. A. (2004). A handbook of reflective and experimental learning. Theory and practice. New York: Routldege Farmer.

Newman, S. y Sánchez,M. (2007). Los programas intergeneracionales: concepto, historia y modelos. En M. Sánchez (Dir.), Programas intergeneracionales. Hacia una sociedad para todas las edades (37-69). Barcelona: Fundación"la Caixa".

Pérez-Serrano, G. (2003). Pedagogía Social. Construcción científica e intervención práctica. Madrid: Narcea.

Puig, J. M., Gijón, M., Martín, X. y Rubio, L. (2011). Aprendizaje-servicio y Educación para la Ciudadanía. Revista de Educación,, 45-67. Recuperado de https://recyt.fecyt.es/index.php/Redu/article/view/11566

Puig, J.M., Martín, X. y Rubio, L. (2017). ¿Cómo evaluar proyectos de aprendizaje servicio?. Voces de la Educación, 2(2), pp.122-132. Recuperado de https://www.revista.vocesdelaeducacion.com.mx/index.php/voces/article/view/72

Real Decreto 1593/2011, de 4 de noviembre, por el que se establece el Título de Técnico en Atención a Personas en Situación de Dependencia y se fijan sus enseñanzas mínimas. BOE, núm. 301, de 15 de diciembre de 2011.

Romero, S. y Torrebadella, X. (2018). CoRubrics: Una evaluación innovadora para una escuela innovadora. Revista Practicum, 3(2), 77-94. Recuperado de https://revistapracticum.com/index.php/iop/article/view/48

Rubio, L., Puig, J. M., Martín, X., y Palos, J. (2015). Analizar, repensar y mejorar los proyectos: Una rúbrica para la autoevaluación de experiencias de Aprendizaje-Servicio. Profesorado, Revista de currículum y formación del profesorado, 19(1), 111-126. Recuperado de https://recyt.fecyt.es/index.php/profesorado/article/view/41025

Salazar, M. y Castro, M.J. (2019). Soledad y convivencia intergeneracional: resignificar el cuidado en el ciclo vital. Revista Reflexiones, 98(2), 79-93. DOI: 10.15517/rr.v98i2.34152.

Santos-González, M.C. y Sarceda-Groso, M.C. (2017). Estructura valorativa de la motivación del alumnado de Formación Profesional hacia sus prácticas curriculares. Revista Prácticum, 2(1),22-39. Recuperado de https://revistapracticum.com/index.php/iop/article/view/18

Saz, Isabel y Ramo, R.M. (2015). Aproximación a los impactos y beneficios del aprendizaje servicio en la Universidad de Zaragoza. RIDAS, 1, 9-27. DOI:10.1344/RIDAS2015.1.2

Solís, M.G. y Mayor, D. (en prensa). Estudio longitudinal comparado del primer nivel del impacto de las Jornadas de Aprendizaje-Servicio en Extremadura. Evaluación de la percepción de los profesionales educativos (2018-2019). Manuscrito entregado para la publicación.

Solís, M.G., Lozano, M., Galán, M.J. y Borrachero, C. (2019). La promoción del Aprendizaje-Servicio en Extremadura. Aprender haciendo sociedades más inclusivas, cooperativas, solidarias y sostenibles. En Varios autores (Coords.), La Convención sobre los derechos del niño a debate 30 años después (pp.901-920). Huelva: CIPI Ediciones. Recuperado de http://www.cipinfancia.org/images/pdf/La CDN a debate 30 aos despus compressed.pdf

Tarabini, A., Jacovkis, J. \& Montes, A. (2017) Los factores de la exclusión educativa en España: Mecanismos, perfiles y espacios de intervención. Project Report. Madrid: UNICEF. 
Tejada, J. (2013). La formación de las competencias profesionales a través del aprendizaje servicio. Cultura y Educación, 25(3), 285-294. DOI: doi:10.1174/113564013807749669.

Traver, J.A., Sales, A. y Moliner, O. (2018). El Aprendizaje-Servicio desde una orientación sociocomunitaria. En O. Chiva Bartoll y M. Pallarès Piquer (Coords.), Aprendizaje-servicio: pasaporte para un futuro mejor (pp. 19-34). Sevilla: Ediciones Egregius.

UNESCO (2016). Educación 2030. Declaración de Incheón y Marco de Acción para la realización del Objetivo de Desarrollo sostenible 4: Garantizar una educación inclusiva y equitativa de calidad y promover oportunidades de aprendizaje permanente para todos. París: UNESCO.

UNESCO (2017). Educación para los Objetivos de Desarrollo Sostenible. Objetivos de aprendizaje. París: UNESCO. 